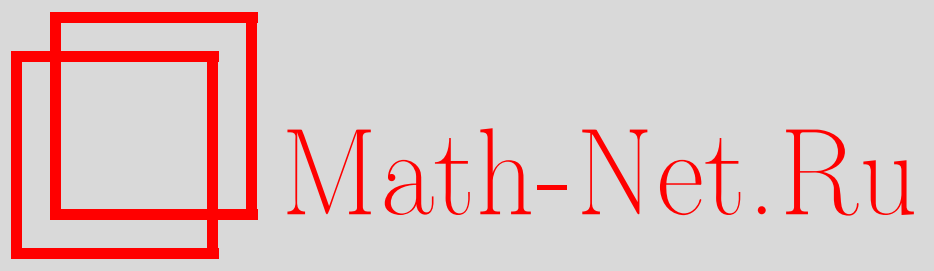

М. Джианфреда, Д. Ландольфи, М. Дж. А. Парис, Проблема квантовой фазы для систем гармонического осциллятора и зависящего от времени осциллятора, $T M \Phi$, 2009, том 160, номер 1, 59-68

DOI: https://doi.org/10.4213/tmf6378

Использование Общероссийского математического портала Math-Net.Ru подразумевает, что вы прочитали и согласны с пользовательским соглашением http://www . mathnet.ru/rus/agreement

Параметры загрузки:

IP : 54.164 .48 .24

26 апреля 2023 г., $15: 38: 47$

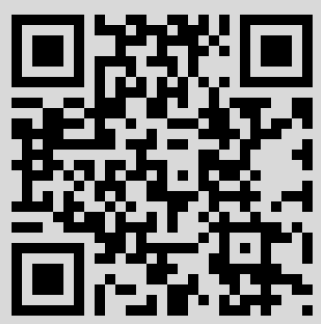




\section{ПРОБЛЕМА КВАНТОВОЙ ФАЗЫ ДЛЯ СИСТЕМ ГАРМОНИЧЕСКОГО ОСЦИЛЛЯТОРА И ЗАВИСЯЩЕГО ОТ ВРЕМЕНИ ОСЦИЛЛЯТОРА}

Рассматриваются обобщенные измерения линейных мультимодовых операторов и обсуждается ряд аспектов, относящихся к построению операторов угла для произвольных квадратичных гамильтоновых систем путем вейль-упорядоченных разложений в терминах операторов координаты и импульса.

Ключевые слова: квантовый оператор угла, обобщенное измерение, система гармонического осциллятора, линейный мультимодовый оператор, гетеродинная регистрация.

\section{1. ВВЕДЕНИЕ}

Построение обладающих хорошим поведением квантовых операторов фазы для гармонических систем является интригующей и сложной проблемой с самого начала развития квантовой механики; эту проблему пытались решить с использованием самых разных физических и математических подходов (см., например, [1]-[3]). Попытки удовлетворительного определения эрмитова оператора, удовлетворяющего каноническим коммутационным соотношениям с оператором Гамильтона (или, в общем случае, с оператором действия), привели к возникновению нескольких вопросов. Довольно быстро стало понятно, что существуют фундаментальные проблемы на пути к этой цели, особенно в свете предложения Дирака использовать схожее с полярным разложение для операторов мод с целью определения квантовых эрмитовых амплитуд и фаз, близких к их классическим аналогам, являющимся комплексными числами, согласно предписанию $\hat{a}=e^{i \hat{\phi}} \widehat{N}^{1 / 2}$, где $\widehat{N}=\hat{a}^{\dagger} \hat{a}$ [4]. Помимо необходимости учитывать периодичность фазовой переменной (свойство, которое несложно было бы установить, если выбрать область определения оператора по модулю $2 \pi$ и ввести $\delta$-функции для того, чтобы базисные канонические коммутационные соотношения выполнялись всюду, за исключением одной границы области определения), встречается еще одно препятствие, состоящее в том, что спектр оператора числа квантов $\widehat{N}$

*Dipartimento di Fisica, Universitá del Salento, Lecce, Italy. E-mail: landolfi@le.infn.it

${ }^{\dagger}$ Dipartimento di Fisica, Università di Milano, Milano, Italy; CNISM, UdR Milano, Milano, Italy; Institute for Scientific Interchange Foundation, Torino, Italy. 
не продолжается на отрицательные значения и оператор $e^{i \hat{\phi}}$ в действительности не унитарен. Более того, невозможно должным образом поделить обе части оператора типа $\hat{a}=\hat{u} \widehat{N}^{1 / 2}$ на $\widehat{N}^{1 / 2}$. Можно было бы вместо этого использовать $\left(\widehat{N}^{-}\right)^{1 / 2}$, где оператор $\widehat{N}^{-}=\sum_{n=1}^{\infty} n^{-1}|n\rangle\langle n|$ является псевдообратным к $\widehat{N}$.

При рассмотрении путей преодоления запрета Паули (т.е. ситуации, когда не существует самосопряженного оператора, канонически сопряженного гамильтониану, если спектр гамильтониана ограничен снизу) было предложено и исследовано несколько концепций кратности квантовой фазы. Среди них имеется подход, основанный на идее расширить гильбертово пространство так, чтобы в нем присутствовали состояния с отрищательными числами квантов (недостижимые для физических систем). Этот подход был развит в работе [5], где было предложено реализовать эрмитов оператор фазы с помощью унитарного оператора $e^{i \hat{\phi}}=$ $\sum_{n=-\infty}^{\infty}|n\rangle\langle n+1|$. С другой стороны, в работе [6] было выдвинуто предложение работать не с самим оператором, а с его периодическими функциями, вводя при этом анзац $\widehat{E} \equiv(\widehat{N}+1)^{-1 / 2} \hat{a}$ для определения некоторого оператора $\widehat{E}$, играющего роль аналога оператора $e^{i \hat{\phi}}$. При этом подходе явная потеря унитарности для $\widehat{E}$ превращается в сужение области определения в гильбертовом пространстве. Внимание уделялось также определению квантовой фазы в рамках представления когерентных состояний [7].

В настоящей работе мы представим некоторые результаты, полученные при рассмотрении проблемы квантовой фазы с двух различных, хотя и связанных друг с другом точек зрения. Сначала в разделе 2 рассматриваются обобщенные измерения для мультибозонных линейных операторов. Напомним, что большинство недавних успехов в отношении квантовых операторов фазы было стимулировано исключительно важным замечанием о том, что самосопряженность в действительности налагает на концепцию наблюдаемой ограничение, не являющееся необходимым: квантовые наблюдаемые являются, вообще говоря, положительными операторнозначными мерами [8], и фазовые наблюдаемые определяются как положительные операторнозначные меры, преобразующиеся ковариантно при временны́х трансляциях [9]. В этой картине постулат квантовой механики о том, что измерению подлежат только наблюдаемые, соответствующие самосопряженным операторам, сохраняется в силу дилатационной теоремы Наймарка. При работе с квантовой переменной, для которой положительная операторнозначная мера не сводится к проекторнозначной мере, различные измерительные системы всегда дадут различные результаты. Так обстоит дело при рассмотрении фазы, и здесь отказываются от концепции идеального оператора квантовой фазы и вводят операторы, связанные с возможными (feasible) квантовыми фазами, определение которых основано на использовании процессов измерения ${ }^{1)}$.

Раздел 2 нашей статьи основан на том, что формулировка проблемы квантовой фазы может опираться даже на использование распределений в фазовом пространстве, которые могли бы дать квантовые средние в виде, напоминающем классические средние. Всякий эрмитов оператор фазы $\hat{\phi}$, для которого фазовое распре-

1) Тем не менее можно определить каноническую переменную фазы [10]. 
деление $P(\varphi)=\operatorname{tr}[\delta(\hat{\phi}-\varphi) \hat{\rho}]$ сопоставляет правильную четко определенную фазу любому локализованному состоянию $\hat{\rho}$ с большой амплитудой, может быть выражен в виде оператора, получаемого из классической фазы путем прямого квантования переменных фазового пространства с добавлением некоторого правила упорядочения, например упорядочения по Вейлю. Подробное обсуждение применений этого подхода к модам стандартного гармонического осциллятора можно найти в работе [11]. Таким образом, естественно возникает вопрос о возможности использования данного подхода всякий раз, когда имеют дело, например, с зависящими от времени осцилляторами. Раздел 3 посвящен некоторым аспектам, следующим из ответа на данный вопрос.

\section{2. ОБОБЩЕННЫЕ ИЗМЕРЕНИЯ И ОПЕРАТОРЫ ФАЗЫ}

Процесс измерения подразумевает взаимодействие между рассматриваемой системой и измерительным прибором. Можно измерять переменную, если она ассоциирована с самосопряженным оператором, определенным в гильбертовом пространстве $\mathcal{H}_{\mathrm{S}} \otimes \mathcal{H}_{\mathrm{P}}$ системы и испытательного устройства. Наблюдатель, живущий в $\mathcal{H}_{\mathrm{S}}$, имеет доступ только к наблюдаемым с носителем в самом $\mathcal{H}_{\mathrm{S}}$. Идея, лежащая в основе обобщенных измерений, состоит в расширении заданных на $\mathcal{H}_{\mathrm{S}}$ операторов переменных до подходящих симметричных операторов в $\mathcal{H}$ : при выполнении ортогонального измерения в $\mathcal{H}$ наблюдатель в $\mathcal{H}_{\mathrm{S}}$ будет знать только о компонентах состояний в своем пространстве. Операторное описание измерения, ограниченного на $\mathcal{H}_{\mathrm{S}}$, дается в терминах положительной операторнозначной меры, которая получается взятием следа спектральной меры расширенного оператора по степеням свободы испытательного устройства [8].

Простейший и наиболее актуальный в этом отношении пример дается измерениями возможной фотонной фазы в контексте гетеродинной регистрации. Гетеродинная регистрация придумана для того, чтобы выполнять совместные измерения двух сопряженых квадратур поля, получающихся посредством смешивания в светоделителе одномодового сигнального поля номинальной частоты $\omega_{1}$ с локальным осцилляторным полем, частота которого $\omega_{\mathrm{L}}$ слегка сдвинута на величину $\omega_{\mathrm{I}} \ll \omega_{1}$ от частоты сигнала (см., например, монографию [12] $)^{2)}$. Фотодетектор располагается после светоделителя, и выходной фототок фильтруется на частоте $\omega_{\mathrm{I}}$. При стандартной оптической гетеродинной регистрации измерение фильтрованного фототока соответствует реализации квантового измерения нормального оператора $\widehat{Z}_{\mathrm{SW}}=a_{1}+a_{2}^{\dagger}$, где $\hat{a}_{1}\left(\hat{a}_{2}^{\dagger}\right)$ - оператор уничтожения (рождения) фотона для входного (преобразованного) сигнала [13]. Измерение вещественной и мнимой частей выходного фототока обеспечивает одновременное измерение вещественной и мнимой частей оператора $\widehat{Z}_{\mathrm{SW}}$.

2) Для мод поля излучения простейшее двухмодовое взаимодействие дается линейным гамильтонианом смешивания $\widehat{H} \propto\left(a_{1} \hat{a}_{2}^{\dagger}+\hat{a}_{2} a_{1}^{\dagger}\right)$, соответствующим взаимодействию, которое имеет место в линейной оптической среде (светоделителе). 
Таким образом, взаимодействие между входным сигналом и светоделительным аппаратом, во-первых, расширяет гильбертово пространство однофотонного сигнала до двухфотонного пространства $\mathcal{H}_{12}=\mathcal{H}_{a_{1}} \otimes \mathcal{H}_{a_{2}}$ сигнала и его образа, а во-вторых, позволяет рассматривать состояния с "отрицательным числом" фотонов. Спектр оператора относительного числа квантов $\widehat{N}_{12}=\hat{a}_{1}^{\dagger} \hat{a}_{1}-\hat{a}_{2}^{\dagger} \hat{a}_{2}$ в действительности неограничен, и на $\mathcal{H}_{12}$ существует унитарный оператор $\widehat{D}_{12}=e^{i \hat{\phi}_{12}}$, удовлетворяющий соотношению $\left[\widehat{D}_{12}, \widehat{N}\right]=\widehat{D}_{12}$. Этот оператор можно записать в виде [14]

$$
\left.\widehat{D}_{12}=\sum_{m=0}^{\infty} \sum_{n=-\infty}^{\infty}|n-1, m\rangle\right\rangle\langle\langle m, n|,
$$

где $|n, m\rangle\rangle$ обозначает базисный элемент в представлении относительного числа состояний, т.е.

$$
\begin{aligned}
|n, m\rangle\rangle & =\Theta(n)|m+n\rangle_{a_{1}}|m\rangle_{a_{2}}+\Theta(-n-1)|m\rangle_{a_{1}}|m-n\rangle_{a_{2}}, \\
\left.\widehat{N}_{12}|n, m\rangle\right\rangle & =n|n, m\rangle\rangle,
\end{aligned}
$$

где $-\infty<n<\infty, m \geqslant 0, \Theta(n)=1$ при $n \geqslant 0$ и $\Theta(n)=0$ при $n<0$. Поскольку $\left[\widehat{Z}_{\mathrm{SW}}, \widehat{Z}_{\mathrm{SW}}^{\dagger}\right]=0$, можно выполнить совместное измерение вещественной и мнимой частей оператора $\widehat{Z}_{\mathrm{SW}}$, что дает реализацию оператора возможной фазы Шапиро-Вигнера. Проблема измерения фазы таким образом сформулирована как оценка фазовых сдвигов, претерпеваемых квантовыми состояниями сигнала и его образа.

2.1. Операторы фазы на гетеродинной основе. Совсем иная ситуация возникает, если частота входного поля лежит за пределами оптического режима и взаимодействие между сигналом и светоделителем приводит к оператору, не являющемуся нормальным [15]:

$$
\widehat{Z}_{\gamma}=a_{1}+\gamma a_{2}^{\dagger}, \quad \gamma=\sqrt{\frac{\omega_{1}-\omega_{\mathrm{I}}}{\omega_{1}+\omega_{\mathrm{I}}}}<1, \quad\left[Z_{\gamma}, Z_{\gamma}^{\dagger}\right]=1-\gamma^{2} .
$$

Коммутатор между операторными квадратурами измерения не обращается в нуль, $\left[\operatorname{Re} \widehat{Z}_{\gamma}, \operatorname{Im} \widehat{Z}_{\gamma}\right]=i\left(1-\gamma^{2}\right) / 2$, поэтому для их совместного измерения следует придумать другой аппарат. Для этого ищется расширение Наймарка оператора $\widehat{Z}_{\gamma}$. Простейшее из таких расширений включает в себя одну дополнительную бозонную моду, скажем $\hat{a}_{3}$, и реализуется с помощью оператора

$$
\widehat{Z}_{\mathrm{N}}=\widehat{Z}_{\gamma}+\kappa \hat{a}_{3}^{\dagger},
$$

определенного на трехмодовом гильбертовом пространстве $\mathcal{H}=\mathcal{H}_{a_{1}} \otimes \mathcal{H}_{a_{2}} \otimes \mathcal{H}_{a_{3}}$ и удовлетворяющего требованиям $\operatorname{Tr}_{a}\left(\sigma a_{3}^{\dagger}\right)=0$ и $\kappa^{2}=1-\gamma^{2}$. Его можно построить, используя билинейные взаимодействия между модами и после этого измерение квадратур на выходе [16]. Оптические приборы ассоциируются с операторами эволюции вида

$$
\widehat{B}_{j k}\left(\theta_{j k}\right)=e^{-i \theta_{j k}\left(a_{j} a_{k}^{\dagger}+a_{k} a_{j}^{\dagger}\right)}
$$




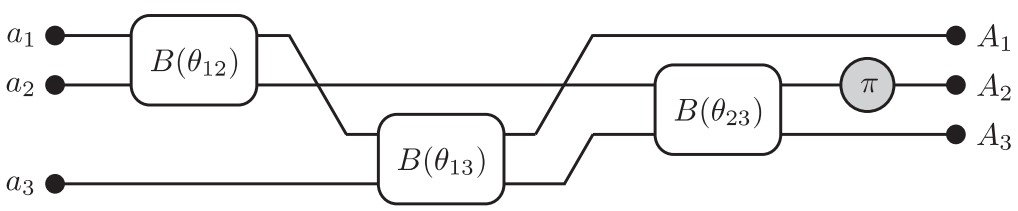

Схема аппарата для обобщенного измерения двухфотонного линейного оператора, не являющегося нормальным. Квадратуры $\left(\hat{A}_{1}+\hat{A}_{1}^{\dagger}\right) / \sqrt{2}$ и $i\left(\hat{A}_{2}^{\dagger}-\hat{A}_{2}\right) / \sqrt{2}$ выходных мод подлежат измерению.

с трехмодовым делением, задаваемым параметрами

$$
\theta_{12}=\arcsin \frac{\gamma}{\sqrt{1+\gamma^{2}}}, \quad \theta_{13}=\arcsin \sqrt{\frac{1-\gamma^{2}}{2}}, \quad \theta_{23}=\arcsin \sqrt{\frac{1-\gamma^{2}}{1+\gamma^{2}}},
$$

которые далее подвергаются повороту на $\pi$ (см. рисунок).

Таким образом, мы видим, что возможную фазу $\hat{\theta}_{\mathrm{N}}$ для квазимонохроматического сигнала вполне можно определить в рамках данного Кейвсом описания гетеродина при условии, что для получения оператора измерения (2.5) вводится мода Наймарка. Эта фаза $\hat{\theta}_{\mathrm{N}}$ получается из однозначного полярного разложения оператора (2.5). Коль скоро введен оператор разности числа квантов для трех мод оператора $\widehat{N}=$ $\hat{a}_{1}^{\dagger} \hat{a}_{1}-\hat{a}_{2}^{\dagger} \hat{a}_{2}-\hat{a}_{3}^{\dagger} \hat{a}_{3}$, коммутатор $\left[\hat{\theta}_{\mathrm{N}}, \widehat{N}\right]=i$ можно интерпретировать как каноническое сопряжение возможной фазы Кейвса $\hat{\theta}_{\mathrm{N}}$ по отношению к $\widehat{N}$.

Теперь нам понятно, как можно устроить обобщенное измерение оператора $\widehat{Z}_{\gamma}$; далее следует проанализировать, какую роль играют приготовления состояний в статистике измерений при проведении конкретных экспериментов. Очевидно, в эксперименте можно в полной мере использовать допустимый произвол в приготовлении некоторых из мод. Подробности по поводу вычисления плотности вероятности на выходе при заданном начальном приготовлении $R_{12} \otimes \rho_{3}$ можно найти в работе [16]. Здесь мы просто отметим, что измерение оператора $\widehat{Z}_{\gamma}$ на классе факторизованных сигналов вида $R_{12}=\varrho_{1} \otimes|0\rangle_{2}\langle 0|$, где $\varrho_{1}-$ приготовление моды $a_{1}$ в общем положении, а $|0\rangle_{2}$ - основное состояние моды $a_{2}$, не приводит к добавлению шума по сравнению с измерением нормального оператора $\hat{a}_{1}+\hat{a}_{2}$, а именно измерения квадратур поля сигнала в рамках стандартной оптической гетеродинной регистрации.

2.2. Мультибозонные линейные операторы. Аргументы, использованные выше для одной моды, можно распространить, по крайней мере в принципе, на системы общего вида. При этом для практической реализации одновременной регистрации сопряженных переменных требуется закодировать расширения Наймарка в устройстве эксперимента. Основную роль в квантовой теории информации играют взаимодействия, линейные и билинейные по модам полей, и их можно экспериментально реализовать в оптических и конденсированных системах. Поэтому на очереди стоит получение ясной картины возможных экспериментальных схем, позволяющих осуществить обобщенные измерения квадратур. Обобщенные измерения 
мультимодовых линейных операторов

$$
\widehat{Z}^{\left(m_{1}, m_{2}\right)}=\sum_{k_{1}=1}^{m_{1}} A_{k_{1}} \hat{a}_{k_{1}}+\sum_{k_{2}=1}^{m_{2}} B_{k_{2}} \hat{a}_{m_{1}+k_{2}}^{\dagger}
$$

где

$$
\left[\widehat{Z}^{\left(m_{1}, m_{2}\right)}, \widehat{Z}^{\left(m_{1}, m_{2}\right) \dagger}\right]=\sum_{k_{1}=1}^{m_{1}}\left|A_{k_{1}}\right|^{2}-\sum_{k_{2}=1}^{m_{2}}\left|B_{k_{2}}\right|^{2} \neq 0,
$$

обсуждаются в работе [17]. Наиболее экономный выбор для допустимого расширения Наймарка $\widehat{Z}_{\mathrm{N}}^{\left(m_{1}, m_{2}\right)}$ снова состоит, очевидно, в добавлении к $(2.7)$ всего одной моды посредством или оператора уничтожения, или оператора рождения, как это диктуется знаком коммутатора $(2.8)$. В силу условия $\left[\widehat{Z}_{\mathrm{N}}^{\left(m_{1}, m_{2}\right)}, \widehat{Z}_{\mathrm{N}}^{\left(m_{1}, m_{2}\right) \dagger}\right]=0$ наблюдаемую возможную фазу $\hat{\theta}^{\left(m_{1}, m_{2}\right)}$ для $\widehat{Z}^{\left(m_{1}, m_{2}\right)}$ можно без опасений определить как фазу одномодового расширенного оператора $\widehat{Z}_{\mathrm{N}}^{\left(m_{1}, m_{2}\right)}$. Оператор $\hat{\theta}^{\left(m_{1}, m_{2}\right)}$ канонически сопряжен $\left(m_{1}+m_{2}+1\right)$-модовому оператору относительного числа квантов

$$
\widehat{N}=\sum_{k=1}^{m_{2}+1} \widehat{N}_{m_{1}+k}-\sum_{k=1}^{m_{1}} \widehat{N}_{m_{1}+k}, \quad \widehat{N}_{r}=a_{r}^{\dagger} a_{r}
$$

Аналогично ранее рассмотренному случаю можно реализовать схему линейного усилителя для обобщенного измерения путем обработки всех мод (входной моды и моды Наймарка), которые линейно связаны с исходными, чтобы получить выходной сигнал, переносимый другим набором мод. Моды $\hat{a}_{k}$ взаимодействуют через унитарный оператор $\widehat{U}^{\left(m_{1}, m_{2}\right)}$, который осуществляет линейное преобразование

$$
\left(\begin{array}{c}
\hat{A}_{1} \\
\vdots \\
\hat{A}_{m_{1}+m_{2}} \\
\hat{A}_{m_{1}+m_{2}+1}
\end{array}\right)=\widehat{U}_{\left(m_{1}, m_{2}\right)}^{\dagger}\left(\begin{array}{c}
\hat{a}_{1} \\
\vdots \\
\hat{a}_{m_{1}+m_{2}} \\
\hat{a}_{N}
\end{array}\right) \widehat{U}_{\left(m_{1}, m_{2}\right)}=M^{\left(m_{1}, m_{2}\right)}\left(\begin{array}{c}
\hat{a}_{1} \\
\vdots \\
\hat{a}_{m_{1}+m_{2}} \\
\hat{a}_{N}
\end{array}\right)
$$

$\left(\left[\hat{A}_{i}, \hat{A}_{j}^{\dagger}\right]=\delta_{i, j} \hat{1}\right)$. Что касается подлежащих измерению квадратур выходных мод, отождествление последовательности светоделителей, естественно обобщающих оптическую схему на рисунке, осуществляется путем наложения требования разложения (которое завершается вращением на $\pi$ ) оператора $\widehat{U}_{\left(m_{1}, m_{2}\right)}$ :

$$
\widehat{U}_{\left(m_{1}, m_{2}\right)}=\widehat{\mathfrak{B}}_{m+1, m} \widehat{\mathfrak{B}}_{m+1, m-1} \ldots \widehat{\mathfrak{B}}_{m+1,1} \widehat{\mathfrak{B}}_{m, m-1} \widehat{\mathfrak{B}}_{m, m-2} \ldots \widehat{\mathfrak{B}}_{2,1},
$$

где

$$
\widehat{\mathfrak{B}}_{j, k}=\left[\widehat{B}_{j, k}\left(\theta_{j, k}\right) \prod_{s \neq j, k} \otimes \mathbb{I}_{s}\right], \quad \widehat{B}_{j k}\left(\theta_{j k}\right)=e^{-i \theta_{j k}\left(\hat{a}_{j} \hat{a}_{k}^{\dagger}+\hat{a}_{k} \hat{a}_{j}^{\dagger}\right)} .
$$

Например, к схеме, изображенной на рисунке, можно прибегнуть для обобщенного измерения оператора

$$
\widehat{Z}^{(2,0)}=A_{1} \hat{a}_{1}+A_{2} \hat{a}_{2}, \quad A_{1}, A_{2} \in \mathbb{R}_{+},
$$


при условии, что светоделители имеют характеристики

$$
\theta_{21}^{(2,0)}=\arcsin \frac{A_{2}}{\sqrt{A_{1}^{2}+A_{2}^{2}}}, \quad \theta_{31}^{(2,0)}=\frac{\pi}{4}, \quad \theta_{32}^{(2,0)}=\frac{\pi}{2},
$$

но та же последовательность светоделителей позволяет реализовать измерение квадратур оператора $\widehat{Z}^{(2,0) \dagger}(0,2)$-типа, не требующее завершающего вращения на $\pi$. Содержанием дальнейших исследований должен стать поиск возможно более эффективных схем обобщенных измерений фазы, а также схем регистрации с целью выполнения обобщенных измерений для представлющих интерес нелинейных усилителей.

\section{3. ПЕРЕМЕННЫЕ ДЕЙСТВИЕ-УГОЛ ДЛЯ ЗАВИСЯЩИХ ОТ ВРЕМЕНИ ОСЦИЛЛЯТОРОВ}

Как уже отмечалось во введении, для эффективного рассмотрения проблемы определения подходящих операторов квантовой фазы использовались различные физические основания. Несмотря на то что основные концептуальные проблемы не решены и что требуется и далее сохранять осторожность, поскольку нелинейные канонические преобразования координат и импульсов в переменные действие-угол не являются, как правило, биективными ${ }^{3)}$, аргументы и эффективные средства, развитые для стандартного гармонического осциллятора, можно приспособить к более сложным системам, по крайней мере, в принципе. Имеет смысл более подробно рассмотреть случай зависящих от времени осцилляторов с гамильтонианом

$$
H=\frac{p^{2}}{2 m(t)}+\frac{m(t) \omega^{2}(t) q^{2}}{2}
$$

ввиду их широкого распространения в физике. Переменные действие-угол для системы, описываемой данным гамильтонианом, принимают вид

$$
\begin{aligned}
& J=\frac{m}{2 \sqrt{\kappa} \sigma^{2}} q^{2}+\frac{1}{2 \sqrt{\kappa}}\left[\frac{\sigma}{\sqrt{m}} p-\sqrt{m} \sigma q \frac{d}{d t}\left(\ln \frac{\sigma}{\sqrt{m}}\right)\right]^{2}, \\
& \theta=\operatorname{arctg}\left\{\frac{\sigma^{2}}{\sqrt{\kappa}}\left[\frac{p}{m q}-\frac{d}{d t}\left(\ln \frac{\sigma}{\sqrt{m}}\right)\right]\right\}
\end{aligned}
$$

где $\kappa$ - произвольная положительная постоянная, а функция $\sigma(t)$ является решением уравнения

$$
\frac{d^{2} \sigma}{d t^{2}}+\left[\omega^{2}-\frac{d}{d t}\left(\frac{1}{2 m} \frac{d m}{d t}\right)-\left(\frac{1}{2 m} \frac{d m}{d t}\right)^{2}\right] \sigma=\frac{\kappa}{\sigma^{3}}
$$

Явное формальное представление в терминах операторов координаты и импульса для вейль-упорядоченного оператора квантового угла, связанного с зависящим от времени осциллятором, можно получить по аналогии с процедурой, использованной

\footnotetext{
3) Мы предполагаем, что операторы, с которыми мы здесь будем иметь дело, не приводят к неоднозначным интерпретациям.
}

3 Теоретическая и математическая физика, т. 160, № 1, 2009 г. 
в работе [18] для стандартного гармонического осциллятора. Это позволяет найти фундаментальный оператор угла в виде [19]

$$
\hat{\theta}_{\mathrm{W}}(\hat{q}, \hat{p})=\sum_{k=0}^{\infty} \alpha_{-k, k}(t) \widehat{T}_{-k, k}(\hat{q}, \hat{p}), \quad k=0,1,2, \ldots,
$$

где

$$
\begin{gathered}
\alpha_{0,0}=\frac{\Delta}{J_{p p}} \operatorname{arctg} \tilde{J}_{q p}^{-1} \\
\alpha_{-1-2 k, 1+2 k}=\frac{(-1)^{k}}{1+2 k} \frac{\Delta}{J_{p p}}\left[-\frac{\tilde{J}_{q q} \Delta}{1+\tilde{J}_{q p}^{2}}\right]^{1+2 k}{ }_{2} F_{1}\left(-\frac{1}{2}-k,-k, \frac{1}{2},-\tilde{J}_{q p}^{2}\right), \\
\alpha_{-2-2 k, 2+2 k}=(-1)^{k+1} \frac{\tilde{J}_{q p} \Delta}{J_{p p}}\left[-\frac{\tilde{J}_{q q} \Delta}{1+\tilde{J}_{q p}^{2}}\right]^{2+2 k}{ }_{2} F_{1}\left(-\frac{1}{2}-k,-k, \frac{3}{2},-\tilde{J}_{q p}^{2}\right), \\
\Delta=\frac{\sqrt{\kappa}}{2 \sigma^{2} \sqrt{\tilde{J}_{q q}-\tilde{J}_{q p}^{2}}}, \quad J_{p p}=\frac{\sigma^{2}}{2 m \sqrt{k}}, \\
\tilde{J}_{q p}=-m \frac{d}{d t}\left(\ln \frac{\sigma}{\sqrt{m}}\right), \quad \tilde{J}_{q q}=\kappa \frac{m^{2}}{\sigma^{4}}+m^{2}\left(\ln \frac{\sigma}{\sqrt{m}}\right)^{2},
\end{gathered}
$$

и

$$
\widehat{T}_{-n, n}=\frac{1}{2^{n}} \sum_{j=0}^{n}\left(\begin{array}{l}
n \\
j
\end{array}\right) \hat{q}^{j} \hat{p}^{-n} \hat{q}^{n-j} .
$$

Для оператора, связанного с зависящим от времени квантовым углом осциллятора, формулы (3.5)-(3.7) дают его выражение через операторы координаты и импульса (определенные в начальный момент времени). Чтобы прояснить действие оператора $\hat{\theta}_{\mathrm{W}}$ на квантовые состояния, а также выяснить, как на состояния влияет механизм потери когерентности, следует получить картину действия операторов $\widehat{T}_{-n, n}$. Чтобы избежать проблем из-за их несамосопряженности на вещественной прямой, можно потребовать пространственный конфайнмент между двумя точками, скажем, положить $q \in[-\ell, \ell]^{4)}$. Это можно обсуждать в свете недавно осуществленных пересмотров теоремы Паули и концепции канонической сопряженности (см. работу [22]), имея при этом в виду, что самосопряженный оператор, канонически сопряженный оператору координаты в ограниченной области, не является, вообе говоря, единственным. Для свободной частицы в ограниченной области известно, что непротиворечивым выбором будет любой оператор $\hat{p}^{\gamma}=-i \partial_{q}$, область определения которого задается векторами из области определения оператора координаты $\hat{q}$ с квадратично интегрируемыми первыми производными, нормы которых удовлетворяют симметричному граничному условию, например $\phi(-\ell)=e^{-2 i \gamma} \phi(\ell)$ при $\gamma \in[0,1)[22]$. Тогда импульсная спектральная задача немедленно решается, что

\footnotetext{
4) Напомним, что для свободной частицы распределение Кийовски по временам прибытия и оператор времени Ааронова-Бома $\widehat{T}_{-1,1}$ были формально связаны друг с другом как в рамках концепции положительной операторнозначной меры без предположения об ограниченности координаты [20], так и как предел распределения, получаемого ограничением координатной области на конечный вещественный интервал [21].
} 
дает собственные векторы $e^{i p_{\gamma, k} q} / \sqrt{2}$ с собственными значениями $p_{\gamma, k}=\gamma+k \pi$. Для каждого допустимого значения параметра $\gamma$ получается ограниченный и самосопряженный оператор обратного импульса (при $\gamma=0$ расходящийся вклад от собственного значения с нулевым импульсом следует устранить “вручную", как обычно). Из (3.5) можно соответственно получить самосопряженные операторы $\widehat{T}_{-n, n}^{\gamma}$. Для системы, описываемой гамильтонианом (3.1) и заключенной в пределах конечной области изменения координаты, этот результат можно использовать, чтобы определить локальный самосопряженный оператор угла в достаточно малой окрестности начала координат (см., например, работы [23]) при симметричном $(\gamma=0)$, или антисимметричном $(\gamma \neq 0)$ граничном условии для импульса. В этом отношении интересно исследовать свойства операторов $\widehat{T}_{-n, n}^{\gamma}$, которым до сих пор уделялось мало внимания. В координатном представлении каждый из них принимает вид интегрального оператора Фредгольма:

$$
\left\langle q^{\prime}\left|\widehat{T}_{-n, n}^{\gamma}\right| \psi\right\rangle=\int_{-\ell}^{\ell} T_{-n, n}^{\gamma}\left(q^{\prime} \mid q\right) \psi(q) d q .
$$

В силу (3.5) прямое вычисление ядра оператора $T_{-n, n}^{0}$ дает, например,

$$
\begin{aligned}
T_{-n, n}^{0}\left(q^{\prime} \mid q\right)= & \frac{\left[i \pi\left(q+q^{\prime}\right)\right]^{n}}{n !} B_{n}\left(\frac{q-q^{\prime}}{2}\right) \Theta\left(q^{\prime}-q\right)+ \\
& +\frac{\left[-i \pi\left(q+q^{\prime}\right)\right]^{n}}{n !} B_{n}\left(\frac{q^{\prime}-q}{2}\right) \Theta\left(q-q^{\prime}\right),
\end{aligned}
$$

где $B_{n}(x)$ и $\Theta(x)$ обозначают многочлены Бернулли $n$-го порядка и ступенчатую функцию Хевисайда. Спектральная задача для операторов с ядром (3.9) принимает вид линейного дифференциального уравнения порядка $2 n$, общие решения которого (вне зависимости от начальных и граничных условий) таковы:

$$
\begin{aligned}
& \Psi_{-2,2}^{0}(q)=\xi_{2,0}+\sum_{r=1}^{3} \xi_{2, r} q^{r-1} F_{2, r}\left(-\frac{q^{4}}{16 \ell \lambda_{-2,2}^{0}}\right), \\
& \Psi_{-3,3}^{0}(q)=\xi_{3,0}+\sum_{r=1}^{5} \xi_{3, r} q^{r-1} F_{3, r}\left(-i \frac{q^{6}}{216 \ell \lambda_{-3,3}^{0}}\right) \quad \text { и т.д., }
\end{aligned}
$$

где $\xi_{n, m}$ - постоянные, $F_{n, m}$ - функции $(n, 2 n-1)$-гипергеометрического типа, a $\lambda_{-n, n}^{0}-$ собственные значения. Общее рассмотрение спектральной задачи для операторов $\widehat{T}_{-n, n}^{\gamma}$ будет приведено в следующей работе.

\section{Список литературы}

[1] P. Carruthers, M. M. Nieto, Rev. Modern Phys., 40:2 (1968), 411-440; J. Bergou, B. Englert, Ann. Phys., 209:2 (1991), 479-505; R. Lynch, Phys. Rep., 256:6 (1995), 367-436; D. T. Pegg, S. M. Barnett, J. Modern Opt., 44:2 (1997), 225-264.

[2] V. Perinova, A. Luks, J. Perina (eds.), Phase in Optics, World Sci., Singapore, 1998.

[3] D. A. Dubin, M. A. Hennings, T. B. Smith, Mathematical Aspects of Weyl Quantization and Phase, World Sci., Singapore, 2000. 
[4] P. A. M. Dirac, Proc. R. Soc. Lond. Ser. A, 114:767 (1927), 243-265.

[5] D. T. Pegg, S. M. Barnett, Phys. Rev. A, 39:4 (1989), 1665-1675.

[6] L. Susskind, J. Glogower, Physics, 1 (1964), 49-61.

[7] H. Paul, Fortschr. Phys., 22:11 (1974), 657-689.

[8] А.С. Холево, Вероятностные и статистические аспекты квантовой теории, Наука, M., 1980.

[9] P. Lehti, J.-P. Pellonpää, J. Math. Phys., 40:10 (1999), 4688-4698.

[10] M. G. A. Paris, Nuovo Cimento B, 111:9 (1996), 1151-1159; Fizika B, 6:2 (1997), 63-74.

[11] A. Royer, Phys. Rev. A, 53:1 (1996), 70-108.

[12] A. Ferraro, S. Olivares, M. G. A. Paris, Gaussian States in Quantum Information, Napoli Ser. Phys. and Astrophys., Bibliopolis, Napoli, 2005.

[13] J. H. Shapiro, S. S. Wagner, IEEE J. Quantum Electron, 20:7 (1984), 803-813.

[14] M. Ban, Phys. Rev. A, 50:3 (1994), 2785-2787.

[15] C. M. Caves, Phys. Rev. D, 26:8 (1982), 1817-1839.

[16] M. Paris, G. Landolfi, G. Soliani, J. Phys. A, 40:26 (2007), F531-F537.

[17] G. Landolfi, M. G. A. Paris, Generalized measurement of non normal multiboson operators by minimal linear coupling, preprint, 2008.

[18] C. M. Bender, G. V. Dunne, Phys. Rev. D, 40:8 (1989), 2739-2742; 40:10, 3504-3511.

[19] G. Landolfi, J. Phys. A, 41 (2008), 185302.

[20] R. Giannitrapani, Internat. J. Theor. Phys., 36:7 (1997), 1575-1584.

[21] E. A. Galapon, F. Delgado, J. Gonzalo Muga, I. Egusquiza, Phys. Rev. A, 72:4 (2005), 042107.

[22] E. A. Galapon, Proc. R. Soc. Lond. Ser. A, 458:2018 (2002), 451-472; 458:2027, 2671-2689.

[23] E. A. Galapon, J. Math. Phys., 45:8 (2004), 3180-3215; E. A. Galapon, R. F. Caballar, R. T. Bahague Jr., Phys. Rev. Lett., 93:18 (2004), 180406. 